\title{
NOUVELLE
}

\section{Influence des morphogènes sur la régionalisation de la zone sous-ventriculaire postnatale}

Diane Angonin, Guillaume Marcy, Olivier Raineteau

\author{
Inserm U846, Stem cell and brain research Institute (SBRI), \\ 18, avenue Doyen Lépine, 69500 Bron, \\ France ; \\ université de Lyon, université Lyon 1, \\ 69500, Bron, France. \\ olivier.raineteau@inserm.fr
}

La zone sous-ventriculaire postnatale : une diversité de lignages qui décroît avec le temps

La zone sous-ventriculaire, située dans la paroi des ventricules cérébraux latéraux (Figure 1A), est l'une des principales régions, dans le cerveau des mammifères, à produire de nouvelles cellules après la naissance. Chez la souris, elle contient des cellules souches produisant et renouvelant, tout au long de la vie, deux types de cellules cérébrales: des neurones (les interneurones du bulbe olfactif ${ }^{1}$ ) et des cellules gliales (celles du corps calleux ${ }^{2}$ et du striatum ${ }^{3}$ ). Son activité gliogénique ${ }^{4}$ est maximale dans les trois premières semaines après la naissance. Les cellules gliales générées migrent et se dispersent de façon radiaire dans les régions entourant les ventricules latéraux. Les cellules neuronales non différenciées (appelées les neuroblastes) suivent une autre voie de migration : elles se déplacent le long d'un chemin, appelé voie de migration rostrale, qui les amène jusqu'au bulbe olfactif, où elles se différencient en neurones fonctionnels. Ces neurones ont pour origine deux populations de progéniteurs: des progéniteurs de neurones GABAergiques (progéniteurs qui expriment le facteur de transcription DLX2 [distal-less homeobox 2] et qui génèrent des neurones qui libèrent de l'acide $\gamma$-aminobutyrique [GABA]), qui sont majoritaires, et des progéniteurs de neurones glutamatergiques (progéniteurs

\footnotetext{
${ }^{1}$ Région cérébrale recevant directement l'information des récepteurs olfactifs et impliquée dans son traitement.

${ }^{2}$ Faisceau de fibres connectant les deux hémisphères cérébraux.

${ }^{3}$ Ensemble de noyaux sous-corticaux impliqué dans le contrôle des mouvements ainsi que dans d'autres processus cognitifs.

${ }^{4}$ Génération des cellules gliales.
}

qui expriment le facteur de transcription TBR2 [T-box, brain, 2] et qui génèrent des neurones qui libèrent du glutamate), minoritaires. La diversité des populations neuronales produites par les progéniteurs de la zone sous-ventriculaire est importante et continue d'être le sujet de nombreuses études [1-3].

II existe une relation directe entre les différents types cellulaires produits et la localisation des cellules souches, au sein de la zone sous-ventriculaire, qui leurs donnent naissance [3]. La zone sousventriculaire est ainsi composée d'une mosaïque de microdomaines spécialisés dans la production de populations cellulaires spécifiques. Tous les microdomaines de la zone sous-ventriculaire contribuent à la genèse des différents types d'interneurones GABAergiques, mais ce sont les plus dorsaux (Figure IB) qui produisent des progéniteurs oligodendrocytaires ${ }^{5}$ et des progéniteurs de neurones glutamatergiques. Cette régionalisation est maintenue jusqu'à des stades avancés bien que la neurogenèse et la gliogenèse décroissent avec l'âge. Cette diminution de l'activité germinale de la zone sous-ventriculaire au cours du temps est particulièrement observée pour les populations de progéniteurs dorsaux (progéniteurs glutamatergiques et oligodendrocytaires) qui s'épuisent dans les premiers mois après la naissance.

La zone sous-ventriculaire postnatale possède donc un potentiel germinal important avec une capacité à générer une grande variété d'interneurones ainsi que les différents lignages gliaux.

\footnotetext{
${ }^{5}$ Les oligodendrocytes sont des cellules gliales permettant la génération de myéline.
}

Ce potentiel est conservé du stade embryonnaire au stade adulte bien que la proportion et la diversité de nouvelles cellules générées diminuent avec l'âge.

\section{Origine et maintien}

de la régionalisation de la zone sous-ventriculaire

L'origine embryonnaire des cellules des différentes régions de la zone sous-ventriculaire est multiple. Cette zone fût longtemps considérée comme exclusivement dérivée d'une structure cérébrale embryonnaire appelée éminence ganglionnaire latérale. Des expériences de traçage génétique ont cependant montré que les parois la constituant ont pour origine différentes régions ventriculaires du cerveau embryonnaire en développement ${ }^{6}$. II existe ainsi une continuité entre l'origine embryonnaire des cellules souches et leur spécification au sein des microdomaines de la zone sous-ventriculaire [3-5]. L’hétérogénéité spatiale des cellules souches neurales, au sein de cette zone, serait donc héritée d'étapes précoces lors du développement embryonnaire et serait immuable. Ainsi, les cellules souches issues du mur dorsal ou latéral, maintiennent leur spécificité lorsqu'elles sont isolées in vitro ou lorsqu'elles sont greffées de façon ectopique dans la zone sous-ventriculaire $[6,7]$.

Bien que ces données suggèrent un codage entièrement intrinsèque de la régionalisation de la zone sous-ventriculaire, des études plus récentes révèlent que des

\footnotetext{
${ }^{6}$ Les cellules du mur dorsal de la zone sous-ventriculaire dérivent principalement d'une structure appelée le pallium alors que les cellules du mur latéral dérivent des éminences ganglionnaires médiale et latérale. Les cellules du mur médial dérivent, elles, d'une autre structure embryonnaire appelée le septum subpallial.
} 


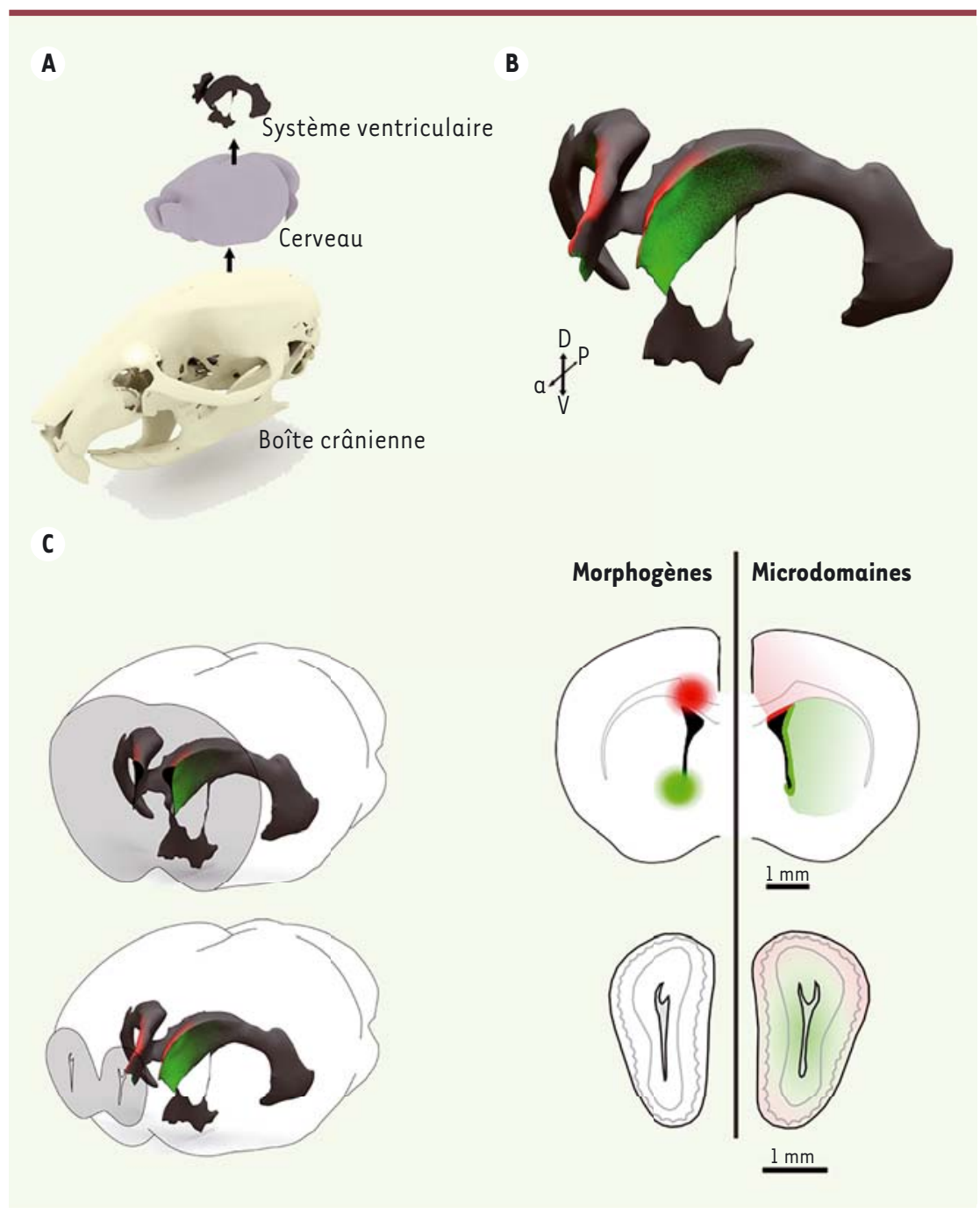

Figure 1. Organisation tri-dimensionnelle de la zone sous ventriculaire. A. Représentation tri-dimensionnelle permettant d'apprécier la taille du système ventriculaire de souris par rapport au cerveau et à la boite crânienne d'une souris adulte. $B$. Représentation tridimensionnelle du système ventriculaire. Les régions germinales dorsale et latérale apparaissent respectivement en rouge et vert. L'activité germinale est maximale dans les régions antérieures du système ventriculaire et diminue dans ses régions les plus postérieures, comme illustré par les gradients rouges et verts qui délimitent respectivement la zone sous ventriculaire dorsale et latérale. C. Localisation de l'activité des morphogènes Wnt (wingless-related integration site) (spot rouge) et Shh (sonic hedgehog) (spot vert), sur l'hémisphère gauche. Représentation schématique des microdomaines dorsaux et latéraux de la zone sous-ventriculaire, sur l'hémisphère droit. La localisation des coupes présentées dans la partie droite est indiquée en grisé sur le cerveau entier dans la partie gauche. Le microdomaine dorsal (ligne rouge) donne naissance à des cellules gliales qui se dispersent de façon radiale dans le corps calleux et dans le cortex sus-jacent (gradient rouge). Le microdomaine latéral (ligne verte) donne naissance à des cellules gliales du striatum (gradient vert). Les deux régions donnent naissance à des neuroblastes qui migrent jusqu'au bulbe olfactif (représenté en bas à droite) où ils se différencient en neurones superficiels ou plus profonds en fonction de leur origine (dorsale ou latérale, respectivement). A : antérieur ; P : postérieur ; D : dorsal ; V : ventral (@Figure élaborée par Aymeric Bianco Pelle et Olivier Raineteau). facteurs extrinsèques participent à son maintien. En effet, l'organisation spatiale de la zone sous-ventriculaire postnatale est maintenue grâce à l'activité de molécules participant à l'identité cellulaire, les morphogènes comme Wnt (Winglessrelated integration site) et Shh (Sonic hedgehog). La manipulation de ces voies de signalisation permet ainsi de changer, au moins partiellement, le devenir des cellules souches qui y résident $[8,9]$.

Un morphogène est une molécule sécrétée qui présente la propriété d'influencer l'identité cellulaire en activant des cascades transcriptionnelles spécifiques. En concentration suffisante dans le milieu, les morphogènes induisent une signalisation intracellulaire (ou voie d'activation), permettant une transduction du signal de la membrane au noyau. Les morphogènes tels que Wnt et Shh ont été très étudiés pour leur rôle dans la mise en place de la polarité de l'embryon (c'est-à-dire son organisation spatiale différentielle le long d'un axe). Leurs implications sont multiples. Elles varient en fonction du stade de développement et du type de cellule sur lesquelles ils agissent. Ces deux familles de morphogènes persistent après le développement embryonnaire. Dans le cerveau postnatal, leur production et leur activité semblent être plus localisées que dans le cerveau embryonnaire, l'organisation cellulaire de la zone sous-ventriculaire postnatale étant plus compacte. Le morphogène Wnt3 est sécrété localement dans la zone par les progéniteurs oligodendrocytaires qui sont présents dans sa région dorsale et dans le corps calleux (Figure 1C). Shh est, quant à lui, produit par des neurones localisés dans la partie ventrale du cerveau antérieur qui possèdent des projections atteignant la zone sous-ventriculaire (Figure IC). Les deux morphogènes sont donc produits par des populations discrètes et distinctes de cellules. Ils semblent avoir également des rôles opposés dans le maintien de la régionalisation de la zone sous-ventriculaire. En effet, des expériences, dans lesquelles chacune des signalisations cellulaires induites par Wnt ou Shh ont été 
suractivées à différents stades du développement postnatal, ont montré que Shh favorise un devenir «ventral» des interneurones produits par la zone sous-ventriculaire (Figure 1C), alors qu'une activation de la voie Wnt promeut la formation de progéniteurs «dorsaux» (Figure 1C), c'est-à-dire oligodendrocytaires et glutamatergiques. Des différences notables ont cependant été observées quant à la capacité de ces deux voies de signalisation à re-spécifier les progéniteurs des régions opposées. Ainsi, une activation de la voie de signalisation Shh dans les régions les plus dorsales de la zone sous-ventriculaire permet la production d'interneurones ayant pour origine des régions plus ventrales [9]. À l'inverse, une activation de la voie de signalisation Wnt dans les régions ventrales ne permet pas de générer des progéniteurs glutamatergiques [8]. Cette tendance des progéniteurs dorsaux à être re-spécifiés peut déjà être observée chez l'embryon et semble refléter une plus grande permissivité des cellules à répondre à la voie de signalisation Shh.

La régionalisation de la zone sous-ventriculaire, bien qu'héritée du développement précoce, n'est donc pas immuable et peut être influencée par la manipulation de morphogènes qui restent actifs après la naissance. Une façon élégante et translationnelle de modifier l'activité de ces différentes voies de signalisation, est l'utilisation de petites molécules pharmacologiques, comme des agonistes de la protéine Smo (smoothened, un récepteur couplé à une protéine $G$ ) qui activent la signalisation Shh, ou des inhibiteurs de l'enzyme GSK3- $\beta$ (glycogen-synthase kinase-3 beta), qui induisent une signalisation Wnt.

\section{Signalétique et plasticité}

phénotypiques des cellules souches postnatales en conditions normales et pathologiques

Bien que les cellules souches aient été proposées comme source potentielle de cellules pour la régénération du système nerveux central postnatal, la compétence de ces cellules (c'est à dire leur capacité de répondre à un signal inducteur) et leur «plasticité » (leur aptitude à changer de devenir) n'ont jusqu'à présent été que peu étudiées. Ces questions sont pourtant primordiales, puisqu'une compétence restreinte de ces cellules limiterait grandement leur potentiel thérapeutique. Cela se traduirait par une capacité réduite de ces cellules à générer (et donc régénérer) une diversité de types cellulaires, et notamment, de sous-types neuronaux. Inversement, la démonstration d'une grande compétence ou d'une plasticité importante, laisserait envisager la possibilité d'une manipulation de ces cellules afin de favoriser leur recrutement dans des conditions pathologiques et leur participation à la régénération tissulaire. Pour le moment, la question reste posée. Des expériences suggèrent une grande plasticité des progéniteurs de la zone sous-ventriculaire tandis que d'autres suggèrent une certaine rigidité du système. Dans tous les cas, une meilleure compréhension de l'organisation de la zone sous-ventriculaire, tant au niveau cellulaire qu'en ce qui concerne la signalétique, est nécessaire afin d'appréhender et d'influencer sa réponse dans une situation pathologique ou lésionnelle.

De nombreuses études suggèrent une contribution de la zone sous-ventriculaire à la régénération du tissu lors de lésions néonatales. II a ainsi été montré que l'hypoxie/ischémie périnatale, responsable d'une perte volumétrique de cortex et de troubles cognitifs à long terme, induit une réactivation de l'oligodendrogenèse et de la neurogenèse corticale. Ces études suggèrent qu'il existe une fenêtre précoce d'opportunité pour le recrutement de cellules nouvellement formées à des fins de régénération tissulaire. Stimuler la prolifération des cellules souches de la zone sous-ventriculaire à l'aide de facteurs mitotiques s'avère bénéfique pour cette régénération et permet d'améliorer la récupération fonctionnelle [10]. Il reste à savoir si cette stimulation se traduit par une déplétion à plus long terme des cellules souches de la zone sous-ventriculaire, ce qui pourrait s'avérer délétère. Une stratégie thérapeutique alternative serait d'utiliser des morphogènes qui permettraient, non pas d'activer les cellules souches de façon globale, mais de favoriser la production ciblée de lignées de cellules, notamment des cellules oligodendrocytaires et neurones glutamatergiques, qui sont particulièrement vulnérables à l'hypoxie. Une meilleure compréhension des mécanismes sousjacents à la régionalisation de la zone sous-ventriculaire et la spécification des différents types cellulaires qu'elle génère, pourrait ainsi permettre l'élaboration de nouveaux outils pour aider à la régénération cellulaire. $\diamond$

\section{Postnatal subventricular zone} regionalization by morphogens

\section{LIENS D'INTÉRÊT}

Les auteurs déclarent n'avoir aucun lien d'intérêt concernant les données publiées dans cet article.

\section{RÉFÉRENCES}

1. Merkle FT, Fuentealba LC, Sanders TA, et al. Adult neural stem cells in distinct microdomains generate previously unknown interneuron types. Nat Neurosci 2014 ; 17 : 207-14.

2. Brill MS, Ninkovic J, Winpenny $\varepsilon$, et al. Adult generation of glutamatergic olfactory bulb interneurons. Nat Neurosci 2009 ; 12 : 1524-33.

3. Mouret A, Lledo PM, Coulombel L. La zone sousventriculaire du cerveau adulte: une mosaïque de cellules souches pré-destinées. Med Sci (Paris) 2008 ; $24: 9-11$.

4. Young KM, Fogarty M, Kessaris N, Richardson WD. Subventricular zone stem cells are heterogeneous with respect to their embryonic origins and neurogenic fates in the adult olfactory bulb. J Neurosci 2007 ; 27 : 8286-96.

5. Fuentealba LC, Rompani SB, Parraguez II, et al. Embryonic origin of postnatal neural stem cells. Cell 2015; $161: 1644-55$.

6. Kelsch W, Mosley CP, Lin CW, Lois C. Distinct mammalian precursors are committed to generate neurons with defined dendritic projection patterns. PLoS Biol 2007 ; 5 : e300.

7. Merkle FT, Mirzadeh Z, Alvarez-Buylla A. Mosaic organization of neural stem cells in the adult brain. Science 2007 ; 317 : 381-4.

8. Azim K, Fischer B, Hurtado-Chong A, et al. Persistent wnt/beta-catenin signaling determines dorsalization of the postnatal subventricular zone and neural stem cell specification into oligodendrocytes and glutamatergic neurons. Stem Cells 2014 ; 32 : 1301-12.

9. Ihrie RA, Shah JK, Harwell CC, et al. Persistent sonic hedgehog signaling in adult brain determines neural stem cell positional identity. Neuron $2011 ; 71$ : 250-62.

10. Scafidi J, Hammond TR, Scafidi S, et al. Intranasal epidermal growth factor treatment rescues neonatal brain injury. Nature $2014 ; 506: 230-4$. 